\title{
Mineração
}

\section{Adsorção de misturas de coletores em silicatos}

\author{
Paulo Roberto de Magalhães Viana \\ Pesquisador Departamento de Engenharia de Minas da EEUFMG \\ E-mail:pviana@demin.ufmg.br \\ Armando Corrêa de Araujo \\ Professor Adjunto Departamento de Engenharia de Minas da EEUFMG \\ E-mail:armando@demin.ufmg.br \\ Antônio Eduardo Clark Peres \\ Professor Adjunto Departamento de Engenharia Metalúrgica da EEUFMG \\ E-mail: aecperes@demet.ufmg.br \\ George Eduardo Sales Valadão \\ Professor Adjunto Departamento de Engenharia de Minas EEUFMG \\ E-mail:gvaladao@demin.ufmg.br
}

\section{Resumo}

Apesar de a prática industrial, na flotação, já considerar a mistura de óleos como "extensores" há mais de 70 anos, somente na década de 60 surgem estudos detalhados sobre interações entre reagentes na flotação. Os primeiros trabalhos dedicaram-se a misturas de reagentes aniônicos ou catiônicos com álcoois. Nos últimos 20 anos, foram publicados alguns trabalhos relacionados à adsorção de misturas de coletores na interface alumina-água, na flotação de feldspato e na flotação reversa de hematita. O presente trabalho revê os principais aspectos da mistura de coletores na flotação e mostra os resultados da microflotação de alguns silicatos com mistura de coletores aniônicos e catiônicos.

Palavras-chave: Flotação, silicatos, adsorção de coletores.

\begin{abstract}
Despite the fact that over 70 years ago the industrial flotation practice already considered the mixture of oils as chain extenders, detailed studies on interactions between flotation reagents appeared only in the sixties. The initial work investigations were dedicated to the study of the mixtures of anionic or cationic reagents with alcohols. Publications during the last 20 years have been related to the adsorption of collector mixtures: at the alumina-water interface, the feldspar flotation, and the reverse flotation of hematite. The present work reviews the main aspects of collector mixture in the flotation and shows the microflotation results of some silicates with the combination of anionic and cationic collectors.
\end{abstract}

Keywords: Flotation, silicate, collector mixture. 


\section{Introdução}

A mistura de diferentes surfatantes, freqüentemente, leva à sinergia de seus efeitos nas propriedades do sistema que se investiga. Esse fato é atribuído ao impacto que a mistura gera na concentração micelar crítica (CMC) e na tensão interfacial (TI), que são menores que a CMC e a TI de cada um dos reagentes considerados isoladamente. Goralczyk (1996) mostra que a diminuição da tensão interfacial (líquido/ar) é dependente da fração molar de cada componente e o maior abaixamento da tensão interfacial é alcançado quando se têm frações equimolares como pode ser visto na Figura 1.

\section{Breve revisão da literatura}

Os cálculos associados à interação de surfatantes seguem o modelo de soluções regulares, porém modelos modificados têm sido sugeridos. Independentemente do modelo adotado, a interação entre os surfatantes é expressa pelo fator $\beta$, cujos valores usuais são mostrados na Tabela 1. Os resultados dos estudos de Huang (1996) indicam associações moleculares menos intensas para o sistema catiônico/não iônico em relação ao aniônico/não iônico.

Os valores obtidos estão em concordância com Scamehorn (1986), que afirma que a atração em sistemas de misturas de reagentes cresce na seguinte ordem: catiônico/não iônico, aniônico/não iônico e catiônico/aniônico.

A adsorção de decilbenzenossulfonato de sódio, na superfície de alumina, na presença de álcool, foi estudada por $\mathrm{Fu}$ et alii (1996). A adsorção foi influenciada, principalmente, pelo comprimento da cadeia do álcool. Para álcool de cadeia curta, propanol, a adsorção de sulfonato decresce e esse fato é explicado pelo aumento da solubilidade do sulfonato na mistura água/propanol. Com álcoois de cadeia maior, a adsorção de sulfonato cresceu e foi maior com o decanol indicando que deve existir um valor crítico para o comprimento da cadeia no qual a adsorção aumenta.

No estudo de Vidyadhar et al.(2003), que trata do feldspato/quartzo com diamina/sulfonato foi demonstrado que até a fração molar 1/1 de diamina/sulfonato obteve-se uma boa resposta na flotação do feldspato e que, quando se excede a

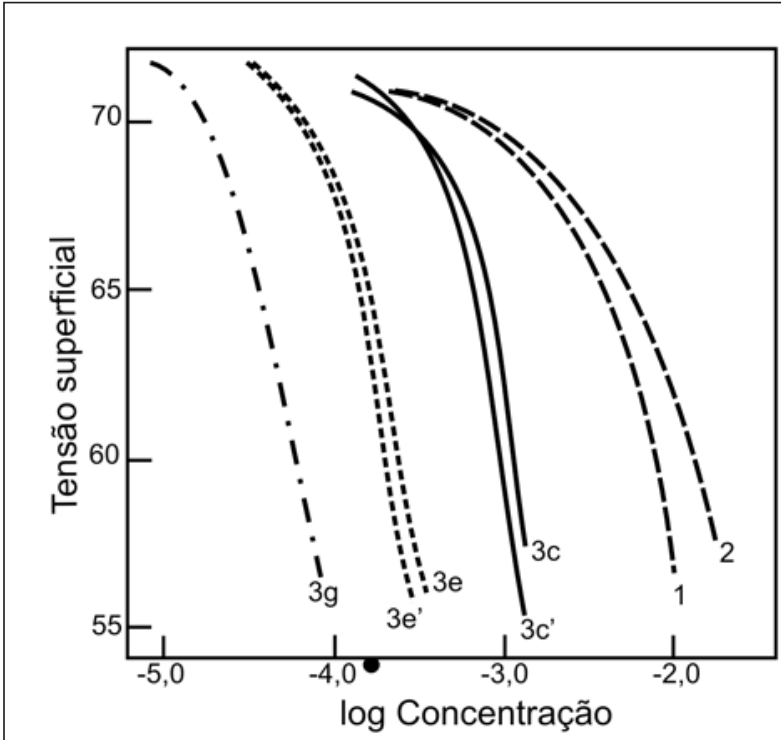

Figura 1 - Efeito sobre a tensão superficial da mistura de surfatantes aniônico e catiônico e tensão superficial da interface líquido/ar. (1) $\mathrm{R}_{10} \mathrm{SO}_{3} \mathrm{Na}$, (2) $\mathrm{R}_{10} \mathrm{P}_{\mathrm{y}} \mathrm{Cl}$, (3) $\mathrm{R}_{10} \mathrm{SO}_{3} \mathrm{Na}+\mathrm{R}_{10} \mathrm{P}_{\mathrm{y}} \mathrm{Cl}$ : $c=1 / 100, c^{\prime}=100 / 1, e=1 / 10, e^{\prime}=10 / 1, g=1 / 1$.

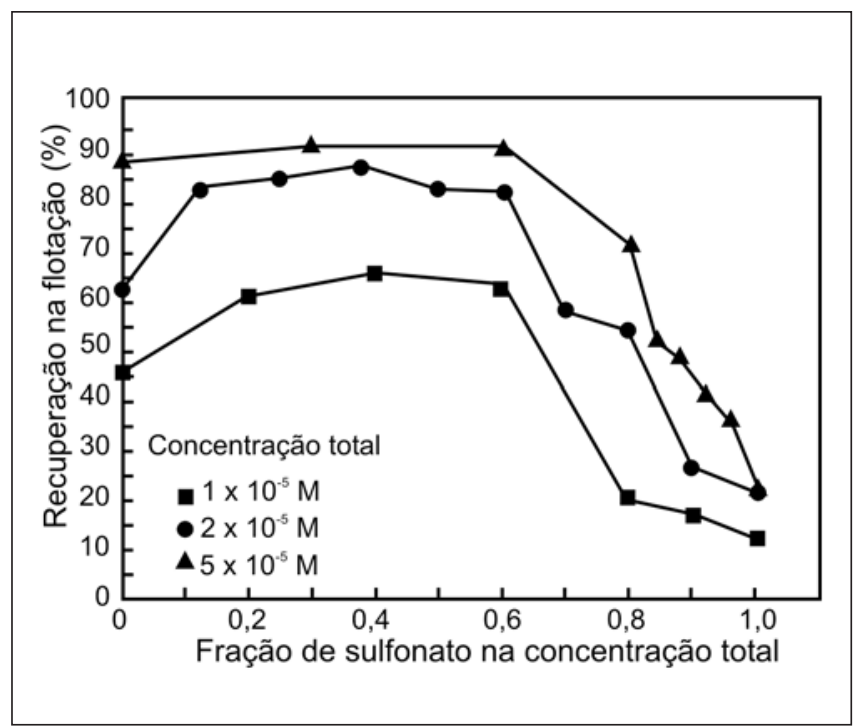

Figura 2 - Sulfonato na flotação catiônica de feldspato. fração $1 / 2$, ocorre a diminuição da flotabilidade, conforme ilustrado na Figura 2.

Silva (2004) estudou a flotação reversa de minério de ferro com amina, na presença de diferentes tipos de álcoois, mostrando que os melhores resultados de recuperação de quartzo estão associados a comprimentos e formas de cadeias de amina e álcool semelhantes, como mostrado na Figura 3.
Tabela 1 - Valores usuais do parâmetro $\beta$.

\begin{tabular}{l|c|c|c}
\hline Natureza do sistema & Valor de $\beta$ & $\begin{array}{c}\text { Huan / } \\
\text { Somasundaran }\end{array}$ & $\begin{array}{c}\text { Somasundaran } \\
\text { / Fu }\end{array}$ \\
\hline Catiônico/aniônico & -25 & & \\
\hline Aniônico/não iônico & -5 a -2 & $-3,5$ & $-1,5$ \\
\hline Catiônico/não iônico & & & \\
\hline Catiônico/catiônico & $-0,2$ & & \\
\hline
\end{tabular}


Paulo Roberto de Magalhães Viana et al.

Helbig et al. (1998) mostram a importância do fator estérico, tanto relativo às cabeças polares, quanto às cadeias hidrocarbônicas, na coadsorção de reagentes aniônicos e catiônicos. Quando existe espaço suficiente entre os grupos polares, é possível a coadsorção de moléculas de um outro reagente, ocorrendo, então, sinergia. Segundo Smith et al. (1973), a mistura de coletores aniônico e catiônico é equivalente a um coletor anfotérico, onde, em faixa neutra de $\mathrm{pH}$, convivem as espécies aniônica e catiônica, levando a um mínimo de solubilidade, capacidade de espumar e hidrofilicidade. Vidyadhar et al.(2002) mostraram que a flotação de quartzo e albita, com o coletor diaminadioleato, apresenta uma resposta equivalente à encontrada para misturas de coletores. O oleato não flota os dois minerais na faixa ácida de $\mathrm{pH}$, porém o coletor anfotérico possibilitou a adsorção de diamina sob a forma iônica e oleato na forma molecular em $\mathrm{pH} 2$.

\section{Flotação de microclina, muscovita, quartzo e espodumênio com misturas de amina, sulfonato, oleato e diaminadioleato}

\section{Procedimento experimental}

As amostras de microclina, quartzo, muscovita e espodumênio mostraram, na difração de raios $\mathrm{X}$, altíssimo grau de pureza. Os testes foram realizados em tubo de Hallimond modificado com volume de $170 \mathrm{~mL}$, usando-se gás nitrogênio com vazão de $20 \mathrm{~mL} /$ minuto e água destilada. A faixa de tamanho dos minerais foi de $-300+106 \mu \mathrm{m}$. Os tempos de condicionamento e coleta foram de 5 minutos e 1 minuto, respectivamente, para 1 grama de mineral. Os principais reagentes, adquiridos junto à Sigma-Aldrich Brasil, tinham grau de pureza de $98 \%$. O coletor diaminadioleato foi fornecido pela Akzo Nobel.

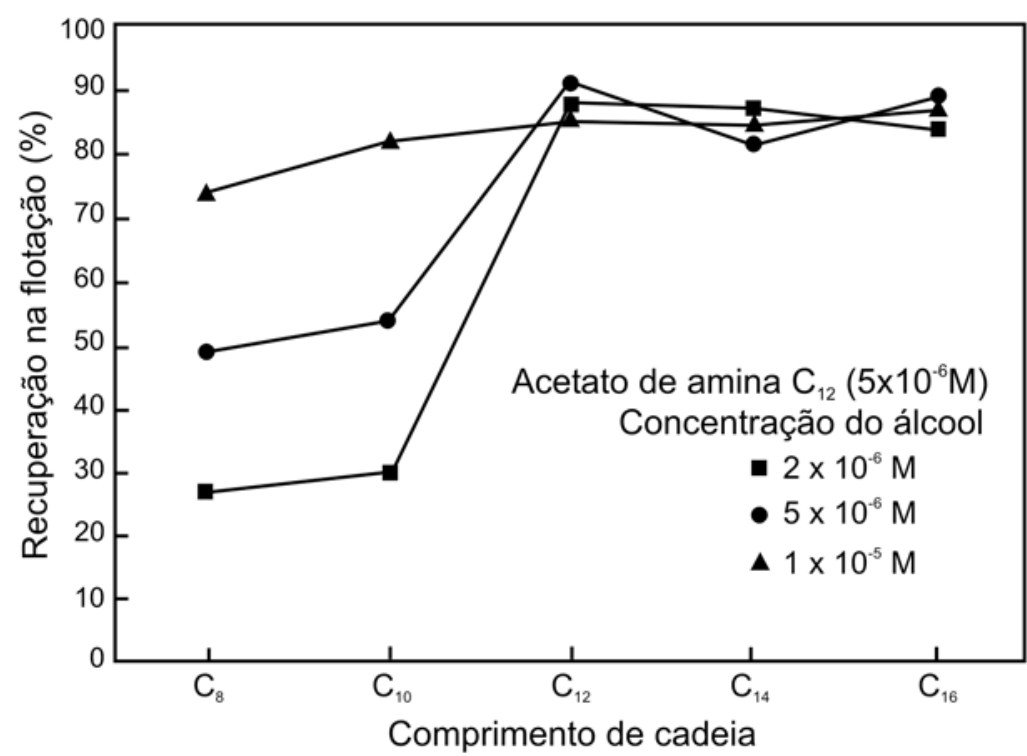

Figura 3 - Influência do comprimento da cadeia do álcool na resposta da flotação.

Resultados obtidos com mistura de amina e sulfonato

Testes anteriores de microflotação do espodumênio, tendo a amina como único coletor, na concentração de $1,0 \times 10^{4} \mathrm{M}$ e pH 5,0, indicaram a possibilidade de flotação reversa do espodumênio. A mistura de amina com sulfonato poderia acentuar as diferenças de flotabilidade ou levar à economia de reagentes. Nos testes preliminares, usando-se somente dodecilamina, na concentração de $3,5 \times 10^{-5} \mathrm{M}$, o quartzo, a microclina e a muscovita mostravam flotabilidades de $30 \%, 60 \%$ e $35 \%$, respectivamente. Verifica-se, agora, na Figura 4(A e B), que o efeito do dodecilsulfonato aumenta a flotabilidade da microclina para $80 \%$ e, praticamente, não altera a flotabilidade do quartzo, que alcança $32 \%$. O efeito é máximo, quando a proporção relativa dos dois reagentes é de $0,9 / 0,1$, sendo muito pequeno, quando a proporção é de 1/1. Uma vez que, em pH 5,0, os dois reagentes estão quase que totalmente dissociados, com cargas de grupos funcionais opostas, o máximo encontrado na proporção de $0,9 / 0,1$ pode ser atribuído à melhor condição de coadsorção entre espécies moleculares neutras, geradas como produto da interação entre os dois reagentes e os íons de dodecilamina. Uma indicação da consistência desse mecanismo é a baixíssima flotabilidade quando a proporção, entre os dois reagentes, é de 1/1, significando associação quase completa das espécies iônicas com a conseqüente diminuição da adsorção.

A influência do comprimento da cadeia do sulfonato na flotabilidade do quartzo, microclina e muscovita, Figura 5 , mostra uma tendência de maior flotabilidade ao se diminuir a cadeia do sulfonato. A melhor flotabilidade foi obtida com cadeia $\mathrm{C}_{8}$, indicando que as possibilidades estéricas de adsorção são favorecidas em uma situação semelhante ao que foi proposto no modelo de Helbig et al. (1998). As flotabilidades do quartzo, microclina e muscovita aumentam para $40 \%, 85 \%$ e $80 \%$, respectivamente.

\section{Resultados obtidos com mistura de amina e oleato}

Novamente ocorreu um máximo na flotabilidade, quando a proporção relativa dos dois reagentes foi próxima de 0,9/0,1, em pH 5,0, porém o espodumê- 
nio, nessa situação, atingiu $80 \%$ de flotabilidade (Figura 6). A dodecilamina, em $\mathrm{pH}$, está totalmente dissociada, enquanto que o oleato tem espécies iônicas e moleculares. Os outros minerais já mostravam flotabilidades em torno de $30 \%$ a $60 \%$ com o uso somente de dodecilamina, correspondendo à região onde já existem hemimicelas. Estima-se que essa situação faça com que uma pequena adsorção do oleato leve imediatamente à formação de uma monocamada onde ocorre o máximo de flotabilidade. No caso do espodumênio, considera-se que a quimissorção do oleato, nos sítios de carga positiva na superfície, em conjunto com a adsorção de natureza eletrostática da dodecilamina, é que leva ao aumento da flotabilidade.

\section{Conclusões}

O máximo de flotabilidade, em $\mathrm{pH}$ 5,0 , obtido na proporção relativa dos reagentes dodecilamina/dodecilsulfonato de $0,9 / 0,1$, pode melhorar a seletividade na flotação da muscovita e da microclina e, secundariamente, a flotabilidade do quartzo, em relação ao espodumênio. Atribui-se a maior flotabilidade nessa condição à coadsorção entre espécies moleculares, produto da interação entre os dois reagentes, e os íons de dodecilamina. A melhor combinação de cadeias foi $\mathrm{C}_{12} / \mathrm{C}_{8}$ para amina/sulfonato, respectivamente, indicando a existência de fatores estéricos na formação do par. As altas flotabilidades em pH 5,0, obtidas com uma proporção relativa de amina/ oleato de $0,9 / 0,1$, não permitiram a definição de uma eventual janela de separação do espodumênio dos outros minerais.

\section{Referências Bibliográficas}

FU, E., SOMASUNDARAN, P., MALTESH, C. Hydrocarbon and alcohol effects on sulfonate adsorption on alumina. Colloids and Surfaces A: Physicochemical and Engineering Aspects, v. 112, p. 55-62, 1996.

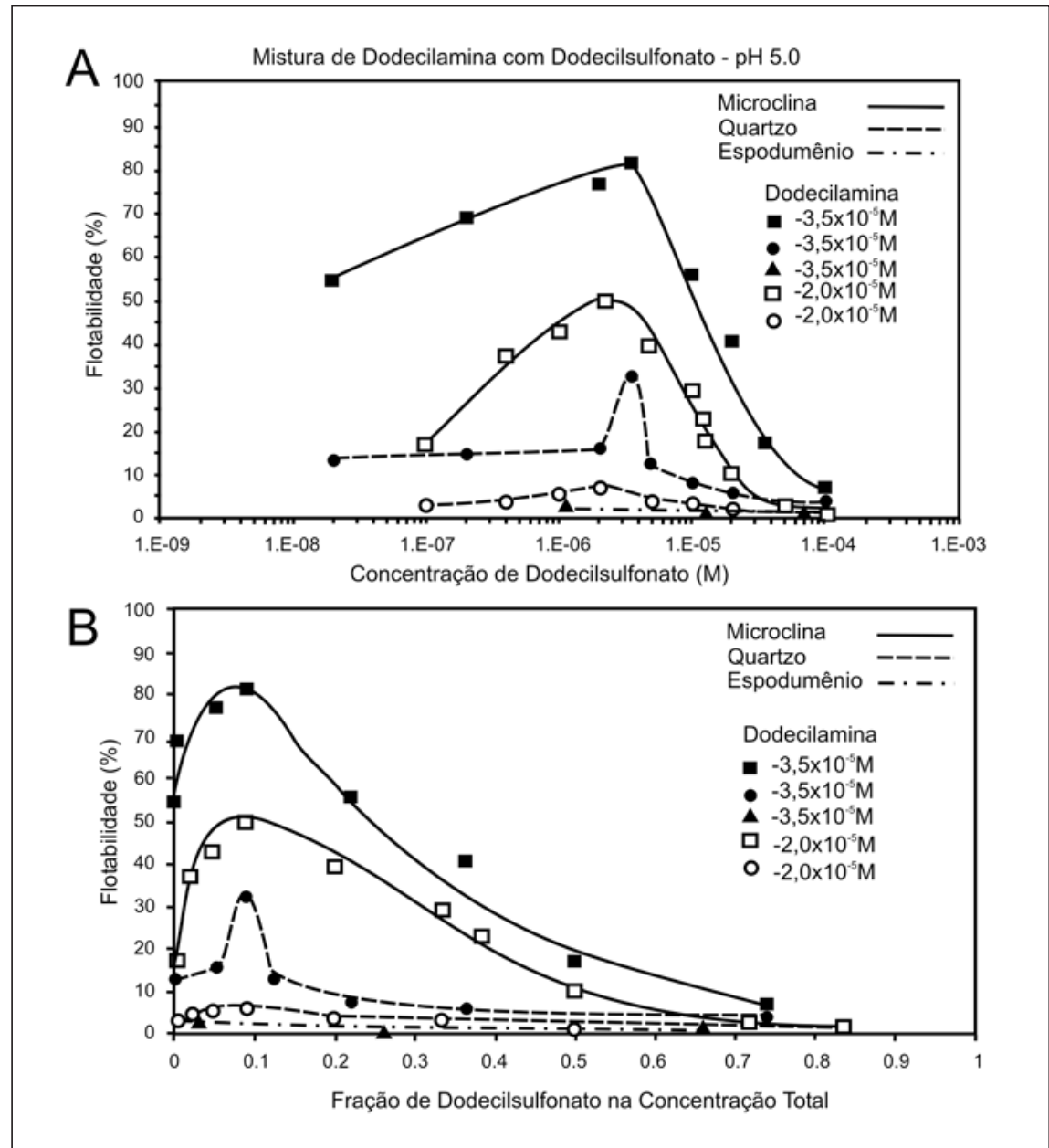

Figura 4 - Flotabilidade de espodumênio, quartzo e microclina com mistura de dodecilamina e dodecilsulfonato.

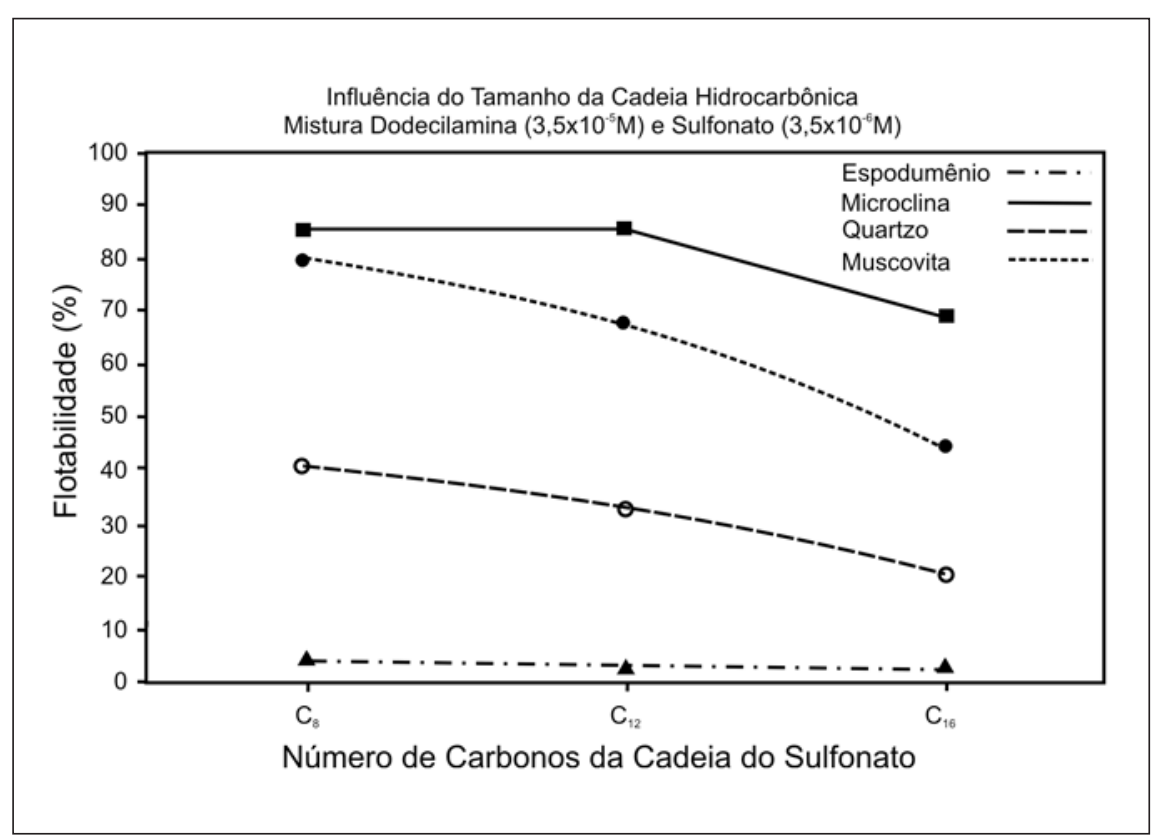

Figura 5 - Influência da cadeia hidrocarbônica do sulfonato na flotabilidade dos minerais estudados. 
Paulo Roberto de Magalhães Viana et al.

GORALCZYK, D. Influence of inorganic electrolyte concentration on properties of anionic-cationic adsorption films. Journal of Colloid and Interface Science, v. 179, p. 211-217, 1996.

HELBIG, C., BALDAUF, H., MAHNKE, J., STOCKELHUBER, K. W., SCHULZE, H. J. Investigation of Langmuir monofilms and flotation experiments with anionic/cationic collector mixtures. International Journal of Mineral Processing, v. 53, p. 135-144, 1998.

HUANG, L., MALTESH, C., SOMASUNDARAN P. Adsorption behavior of cationic and nonionic surfactant mixtures at the alumina-water interface. Journal of Colloid and Interface Science, v. 177, p. 222-228, 1996.

SCAMEHORN, J. F. An overview of phenomena involving surfactant mixtures. In: SCAMEHORN, J. F. Phenomena in mixed surfactant systems. ACS Symposium Series Washington, DC, p. 1-27, 1986

SILVA, R.R.R. Sistemas de reagentes surfatantes na flotação catiônica reversa de minérios de ferro. Escola de Engenharia da UFMG, 2004. 257 p. (Dissertação de Mestrado em Engenharia Metalúrgica).

SMITH, R. W., HADDENHAM, R., SCHROEDER, C. Amphoteric surfactants as flotation collectors. Transactions AIME, New York, v. 254 , p. 231-235, 1973.

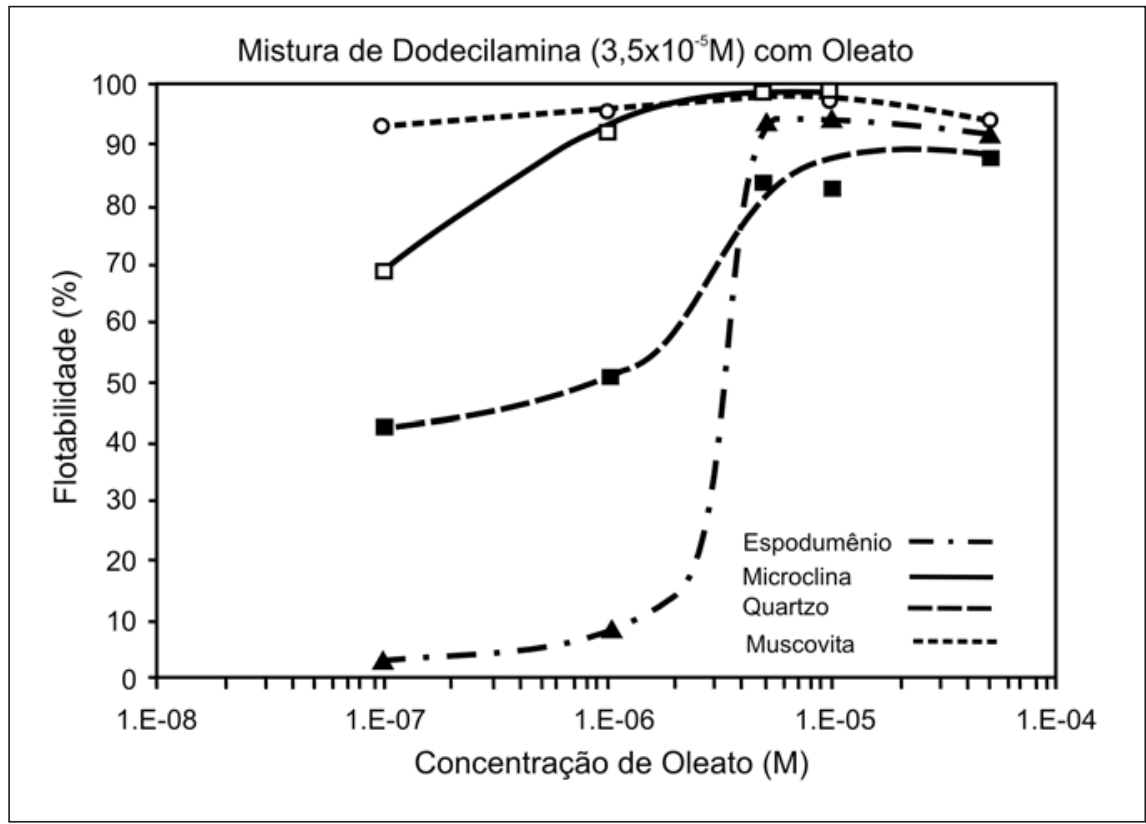

Figura 6 - Flotabilidade de espodumênio, quartzo e microclina com mistura de dodecilamina e oleato.

VIDYADHAR, A., HANUMANTHA RAO, K., CHERNYSHOVA, I. V. Mechanisms of amine-feldspar interaction in the absence and presence of alcohols studied by spectroscopic methods. Colloids and Surfaces A: Physicochem. Eng. Aspects, v. 214, p. 127-142, 2003.

VIDYADHAR, A., HANUMANTHA RAO, K., FORSSBERG, K. S. E. Adsorption of $N$ Tallow 1,3-Propanediamine-dioleate collector on albite and quartz Minerals, and selective flotation of albite from greek stefania feldspar ore. Journal of Colloid and Interface Science, USA, v. 248, p. 19-29, 2002.

Artigo recebido em 01/06/2006 e aprovado em 10/11/2006.

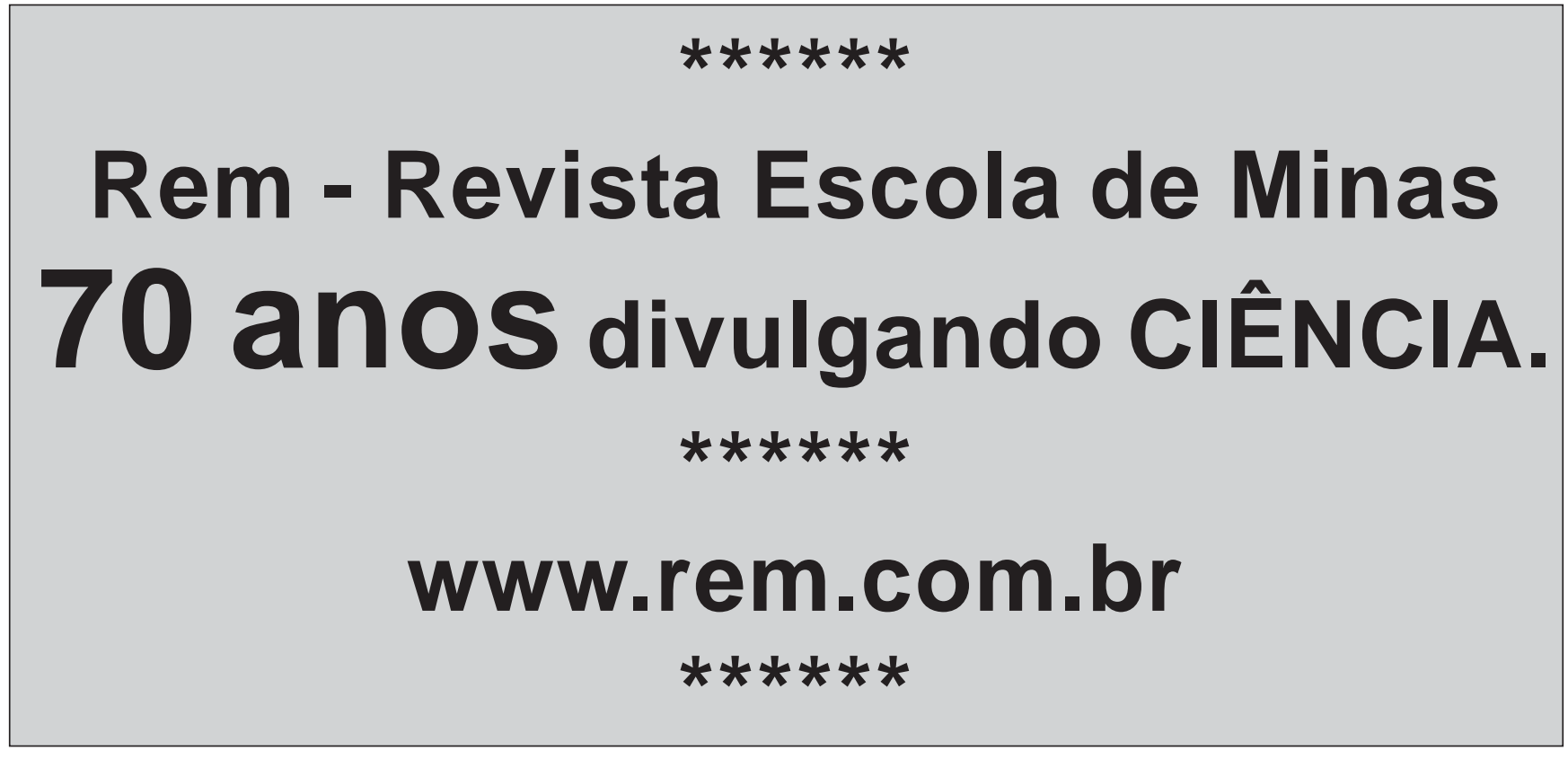

REM: R. Esc. Minas, Ouro Preto, 59(4): 421-425, out. dez. 2006 